\title{
An Approach to Managing Gynecologic Tumors and Precancerous Conditions by Vaginal Surgery
}

\section{Ozlem Ozyilmaz*}

Obstetrics and Gynecology, Department of Urogynecology, Health Sciences University, Dr. Lutfi Kirdar Kartal Training and Research Hospital, Istanbul, Turkey

*Corresponding author: Ozlem Ozyilmaz, Obstetrics and Gynecology, Department of Urogynecology, Health Sciences University, Dr. Lutfi Kirdar Kartal Training and Research Hospital, Istanbul, Turkey, E-mail: drozlemsarici81@gmail.com

Received date: September 01, 2018; Accepted date: October 09, 2018; Published date: October 15, 2018

Copyright: (C) 2018 Ozyilmaz O. This is an open-access article distributed under the terms of the Creative Commons Attribution License, which permits unrestricted use, distribution, and reproduction in any medium, provided the original author and source are credited.

Citation: Ozyilmaz O (2018) An Approach to Managing Gynecologic Tumors and Precancerous Conditions by Vaginal Surgery. Crit Care Obst Gyne. Vol.4 No.3:13

\section{Editorial}

Pelvic organ prolapse (POP), has been described as bulging intra-abdominal organs from vagina including bladder, urethra, uterus, small bowel and rectum. If prolapse of the bladder occurs into the vagina called as cystocele; if prolapse of the urethra occurs called as urethrocele; if prolapse of uterus occurs called as uterine prolapse; if prolapse of small bowel occurs called as enterocele and if prolapse of rectum occurs called as rectocele. Pregnancy, obesity, respiratory problems, constipation, pelvic organ cancers are the main reasons of pelvic organ prolapse due to increased pressure in the abdomen. The patient complains of bulging mass, genital pain, urinary symptoms, constipation, bleeding and sexual dysfunction. Treatment of pelvic organ prolapse includes kege lexercises which depends on building of pelvic flor muscles; mechanical treatments called as pessary to provide support for lifting up bulging organs from vagina; surgical treatment, either to repair the affected structure or organ or to remove the organ [1].

Gynaecologic tumours or disorders (benign or malign) coexistent with pelvic organ prolapse (POP), still has no standardized treatment protocol which has been established yet. The first objective of this commentary, is to highlight the management of some gynaecologic oncologic diseases, which hysterectomy and bilateral salpingo-ooferectomy is enough for them such as endometrium cancers(Stage I-IIA), myomas in different locations (Benign myomas turn into leiomyosarcomas in large myomas, postmenopausal women, rapid growing myomas), postmenopausal uterine polyps causing symptom or not (Endometrial polyps can be detected in addition with endometrium cancer; especially in postmenopausal woman who are bleeding), benign ovarian masses and, preinvasive cervical diseases, in association with POP [2].

Another aim of this commentary is how to add prophylactic bilateral salpingo-ooferectomy to vaginal hysterectomy due to the strong family history independently of BRCA Mutations, in every case including mild POP described as Stage I-II according to Pelvic Organ Prolapse Quantification System (POP-Q). BRACA1 and $B R C A 2$ are the predictor parameters of ovarian and breast cancers. Independently of cancer predictors, the patient can be at high risk because of strong family history. If the patient is at high risk for ovarian cancer should undergone prophylactic bilateral salpingo-ooferectomy even in addition with benign indications of hysterectomy. Hysterectomy in addition with bilateral salpingo-ooferectomy can be performed by vaginally, laparotomy, laparoscopy orlaparoscopy assisted vaginally [3]. Vaginal approach is the best way for all gynaecologic surgeries including oncology. The surgeon can prefer vaginal way instead of laparotomy or laparoscopy. Only a good plan before the operation, and to be good at pelvic flor anatomy is required, then it is possible to do vaginal hysterectomy and bilateral salpingo-ooferectomy in complicated cases with severe POP or mild POP [4].

Another advantage of vaginal approach is vaginal remodelling which provides high quality of sex life according to the Female Sexual Function Index (FSFI). The patient feels well by increased quality of sex life and thinks that she hasn't undergone an operation for oncologic reason, because of the absence of visible abdominal scar. Laparoscopic lymphadenectomy should be added after the pathological evaluation if it is needed in some cancers, so it can be described as laparoscopy assisted vaginal surgery in gynaecologic cancer survivors [5].

\section{Keywords: Oncology; Pelvic organ prolapse; Myomas}

\section{References}

1. Antosh DD, High R, Brown HW, Oliphant SS, Abed H, et al. (2017) Feasibility of prophylactic salpingectomy during vaginal hysterectomy. Am J Obstet Gynecol 217: 1-605.

2. Genc M, Celik E, Eskicioglu F, Bahar Gur E, Kurtulmus S, et al. (2016) The relationship between uterine prolapse and premalignant endometrial pathology. Clin Exp Obstet Gynecol 43: 500-503.

3. Teramoto S, Narushima M, Kojima I, Takagi Y, Shimoji T (2014) Study on the treatment of pelvic organ prolapse complicated with uterine myoma. Nihon Hinyokika Gakkai Zasshi 105: 183-189.

4. Peng J, Sinasac S, Pulman KJ, Zhang L, Murphy J, et al. (2018) The feasibility of laparoscopic surgery in gynecologic oncology for obese and morbidly obese patients. J Gynecol Cancer 28: 967-974. 
5. Nemeth G (2014) Indications and methods of hysterectomy. Orv Hetil 155: 1152-1157. 